\title{
Clinical characteristics of 64 cases of patients infected with novel coronavirus 2019 (COVID-19) in Wenzhou, China
}

\author{
Jichan Shi ${ }^{1}$, Zhijie $\mathrm{Yu}^{2}$, Ziyang Huang ${ }^{2}$, Rongrong $\mathrm{Chen}^{2}$, Yiyi Zhu ${ }^{3}$, Wei Chen ${ }^{3}$, Aiqiong \\ Cheng $^{1}$, Hongye Ning ${ }^{3}$, Yueying Zhou ${ }^{3}$, Xiangao Jiang ${ }^{3}$, and Kang YU ${ }^{4}$ \\ ${ }^{1}$ Wenzhou Central Hospital \\ ${ }^{2}$ The First Affiliated Hospital of Wenzhou Medical University \\ ${ }^{3}$ Affiliation not available \\ ${ }^{4}$ Wenzhou Medical University First Affiliated Hospital
}

May 11, 2020

\begin{abstract}
Abstract: Background: COVID-19 burst in Wuhan China in December 2019 and soon became a worldwide emergency. To help further understand COVID-19. Here we report the epidemiological, clinical features, potential biomarkers, and CT changes of cases in Wenzhou. Methods:All 64 cases were diagnosed by positive of PCR test. Clinical laboratory tests, CT and treatment were performed in the level 3 of protection. Data and clinical characteristics feature were collected by 4 th March. The correlation was analyzed. Results:Most cases were light. The average of latency was 3.4 days, ranged from 1-14. The median age was 44 , ranged from 2.2-67; 36 male and 28 female; 59 had fever, 38 with high temperate (>38); 45 had respiratory symptoms; 28 had gastrointestinal symptoms; 39 weakness; one asymptomatic case was found. Most with low LC, K+, Na+, OI and high LDH, CRP, D-dimer. Pneumonia, both lungs involvement, ground-glass opacity and consolidation were shown by CTs. Minor had electrocardiography change. Conclusions:The clinical features, abnormality lab indexes and CT characteristics of COVID-19 were like SARS, majored in fever, weakness along with dry cough, diarrhea. LC, LDH could be good biomarkers monitoring progress. The elders, hypertension patients and smokers were more vulnerable. COVID-19 was milder, but more transmissible than SARS. Latency could be up to 14 days without symptoms. CT and PCR shall be both considered in clinical high suspicion. There are still further efforts to be made as to preventing and control the epidemic and the cost in world wild.
\end{abstract}

\section{Keywords: COVID-19, LDH, LC, Electrolyte imbalance, Wenzhou}

\section{Introduction}

Coronaviruses are a group of viruses with crown-like spikes on the surface of envelope [1]. Although most of them are common and mild infection, the epidemics of SARS-CoV[2-4] and MERS-CoV [5 6] are still nightmare since 21 century with mortality rates of $10 \%$ for SARS-CoV and $37 \%$ for MERS-CoV[7 8] . In December 2019, China first reported a cluster of cases of unknown pneumonia in Wuhan, and the people infected may be associated with exposure to Huanan seafood market[9]. A novel coronavirus was identified as the pathogen of this outbreak, termed as COVID-19 by the WHO[10]. The epidemics of COVID-19 soon spread to the whole country and cases were reported in other 18 countries make it finally a public health emergency with international concern over global outbreak [11]. However, phyloepidemiologic analyses indicated the SARS-CoV-2 source in China should be imported from other places[12]. So far, the original source has still not been found, than 2.4 million confirmed cases and more than 163000 deaths have been confirmed[13] (reported by WHO, April 22nd,2020).

Wenzhou, a city outside Hubei, has been one of top 10 cities of most cases reported in China since COVID-19 outbroke. To help further understand the novel coronavirus COVID-19 infection and treatment, we describe 
the clinical features of 64 cases infected by COVID-19 in multi-center in Wenzhou (Data accessed till March 4th). In our studies, we found several characterize features have the correlation to the disease severity, which could be good biomarkers to monitor the disease progress.

\section{Materials and methods}

\section{Patients}

64 cases admitted to several hospitals in Wenzhou from $17^{\text {th }}$ Jan to $29^{\text {th }}$ Jan 2020 , were diagnosed, classified and treated recommended by the standards[14 15]. 3 typical cases of different severity were chosen to take an example of disease development. All subjects had signed the informed consents and the study were performed in accordance with guidelines approved by the Ethics Committees from Wenzhou Central Hospital.

\section{Data collection and analysis of clinical findings.}

The data collection including epidemics characterizes, clinical feature information, clinical lab test, chest computer tomographic (CT), electrocardiography and were collected at the earliest time points when hospitalized in the level 3 of biosafety protection.

\section{The criteria of diagnostic, treatment and discharge}

The criteria of diagnostic, treatment and discharge were recommended by the standards published by National Health Commission of the People's Republic of China[14 15]. The definite diagnosis was made by upon the suspected case, having continuous Positive-Realtime PCR confirmation of COVID-19 with an interval more than $24 \mathrm{~h}$ in respiratory samples (sputum or throat swabs) monitored by Wenzhou CDC and were reconfirmed by Zhejiang CDC. Classification: Light, had fever respiratory symptom with pneumonia checked by imaging; Severe, satisfied any of the following, respiratory distress, RR [?] 30 times per min, oxygen saturation [?] 93\% or OI (PaO2/ FiO2) [?] 300 mmHg $(1 \mathrm{mmHg}=0.133 \mathrm{kPa})$; Critical, satisfied any of following, respiratory failure developed, and mechanical ventilation was required, shocked or combined with other organ failure that need ICU care unit. The patients were treated with supporting treatment, isolation ward, rest informed, more water taken, oxygen therapy, strengthen rehydration, correct electrolyte imbalance, close observation and triple antivirus therapy: recombinant human interferon infusion, Lopinavir/ritonavir (Kaletra), Abidor hydrochloride tablets; Chinese traditional treatment was also used, Lianhua qingwen capsule; and other treatments, like antibiotic, glucocorticoid if needed. The discharge was made by normal temperate lasting more than 3 days, better change of respiratory symptom and continuous tests (more than 24h) of COVID-19 PCR were negative.

\section{Statistical analyzes}

All the indexes were described with mean and SD. Single-T test was used to verify if the clinical feature and indexes of patients had significant to healthy population. The spearman correlation coefficient was made between two groups with continuous variables. The P-value less than 0.05 was considered as statistically significant. All tests were taken under bilateral performed with SPSS 19.0.

\section{Results}

All 64 cases were admitted to multi-centers in Wenzhou, such as the first affiliated hospital of Wenzhou Medical University (WMU), The second affiliated hospital of WMU and Wenzhou Central Hospital. Cases were diagnosed, classified and treated recommended by the standards published by National Health Commission of the People's Republic of China[14 15]. All patients were diagnosed by continuous Positive-Realtime PCR confirmation of COVID-19 with an interval more than $24 \mathrm{~h}$ in sputum or throat swabs and were reconfirmed by Zhejiang Center for Disease Control and Prevention (CDC). Clinical laboratory tests, computer tomographic and hospitalization treatment were performed in the level 3 of protection. Data and clinical characteristics feature were collected by $4^{\text {th }}$ March.

\section{Epidemic source:}


In 64 cases, median age was 44, $58(90.63 \%)$ cases were ranged from 20-60, only $5(7.81 \%)$ were above 60 ; a 26 -months infant was found. In gender, 36(56.25\%) were male and $28(43.75 \%)$ were female. There were 62 $(98.44 \%)$ had the clear contact history, 33 patients who had been to Wuhan in last 14 days, were classified as Generation 1 (Gen.1); 25 closely contacted the Gen.1,as Gen.2; 4 were family members of Gen.2, as Gen.3; 2 lost contact history. Importantly, 16 cases were reported from a same place imported by one Gen.1. (Table

\section{1, Figure 1 )}

\section{Clinical features and treatments:}

The main clinical features developed including fever, high temperate, dry cough, weakness, many cases had expectorations, shortness of breath, diarrhea, nausea and vomit; Pharyngalgia and muscular soreness were not obvious; Catarrhal symptoms are rare; the severe and critical patients had varying degrees of shortness of breath and difficulty in breathing (Table 1 ). 1 asymptomatic case without any symptoms was found. $51(79.69 \%)$ were classified as light, while $11(17.19 \%)$ were severe and critical were $2(3.12 \%)$. The mean of latency of were 3.4 days range from 1-14. The maximum temperatures were recorded when hospitalized. It was thought to be fever if the ear temperate was above 37.3. Most cases $(59,92.19 \%)$ had fever, average with 6.9 days, most fevers $(38 / 55,69.1 \%)$ were above 38 , the average temperature was 38.1 .5 cases had no fever, just a little unobvious symptom. More than half $(39,60.9 \%)$ had Weakness, few $(5,7.8 \%)$ had Muscular soreness. Respiratory symptoms were seen in $45(70.3 \%)$ cases, as dry Cough 41 (64.1\%), expectoration 23 (35.9\%), shortness of breath $18(28.1 \%)$, pharyngalgia $10(15.6 \%) .28(43.8 \%)$ cases had gastrointestinal symptoms, 14 (21.9\%) Diarrhea, 13 (20.3\%) Nausea, 8 (12.5\%) Vomit. And there were 15 (23.4\%) secondary infection cases. The basic disease including Hypertension (20) and Chronic liver disease (7). No Smoke of all.

\section{Clinical indexes:}

All cases were PCR positive. The clinical indexes were collected as shown in Table 2 , including WBC, NEC, LC, PLT, Hb, ALT, AST, $\mathrm{K}^{+}, \mathrm{Na}^{+}$, Cr, CK, LDH, TNI, BNP, CRP, OI $\left(\mathrm{PO}_{2} / \mathrm{FiO}_{2}\right)$ and D-dimer. All indexes were described with mean and SD. It was found that WBC, NEC, LC, PLT, Hb, K+, Na+, LDH, CRP, OI (Oxygenation Index) and D-dimer had the statistical significant change $(\mathrm{p}<0.001)$, so did AST $(\mathrm{p}<0.05)$. The decreased indexes were WBC, LC, PLT, K+, Na+, OI, while LDH, CRP, D-dimer would be higher (Figure 2) . ALT, Cr, BNP and TNI were normal.

We further analyzed the correlation between these indexes and clinical features. The severity had no correlation with generation, gender, WBC, NEC, K+, Na+, AST, CRP, PLT, Hb, latency or Fer, but had significant in correlated to age, temperate, LC, LDH, D-dimer, OI (Supplement 1). To make it clear, partial results were picked from Supplement 1 asTable 3 . It was showed that severity had the positive correlation with $\mathrm{D}$-dimer $(\mathrm{R} 2=0.293, \mathrm{p}=0.033)$, and age $(\mathrm{R} 2=0.325, \mathrm{p}=0.009)$, temperate $(\mathrm{R} 2=0.339, \mathrm{p}=0.006), \mathrm{LDH}$ $(\mathrm{R} 2=0.472, \mathrm{p} j 0.001)$, while had negative correlation with $\mathrm{LC}(\mathrm{R} 2=-0.385, \mathrm{p}=0.002 \mathrm{j} 0.01)$ and $\mathrm{OI}(\mathrm{R} 2=-0.712$, pi0.001) (Table 3, Figure 3 ). Besides, age had negative correlation with $\mathrm{K}+, \mathrm{LC}$ and OI; temperate were negative correlated to LC $(\mathrm{R} 2=-0.279, \mathrm{p}=025)$ and $\mathrm{OI}(\mathrm{R} 2=-0.433, \mathrm{p}=0.001)$; D-dimer also had the correlation with $\mathrm{LDH}(\mathrm{R} 2=0.421, \mathrm{p}=0.002)$; OI had negative correlation with most of features and clinical indexes, such as severity $(\mathrm{R} 2=-0.712, \mathrm{p}<0.001)$, age $(\mathrm{R} 2=-0.298, \mathrm{p}=0.021)$, temperate $(\mathrm{R} 2=-0.433, \mathrm{p}=0.001), \mathrm{LDH}$ $(\mathrm{R} 2=-0.552, \mathrm{p}<0.001)$, D-dimer $(\mathrm{R} 2=-0.311, \mathrm{p}=0.023)$, except positive for $\mathrm{LC}(\mathrm{R} 2=0.403, \mathrm{p}=0.001)$.

To find whether there was the difference between patients who were discharged and those still in hospitalization, we also did an independent-samples T test, but the results showed no differences. SeeSupplement Table 2.

\section{Chest CT:}

Almost all $(62,96.9 \%)$ had varying degrees of lung symptoms detected by chest computer tomographic, but there were still two cases normal. The most symptoms changed shown by CT were Pneumonia $(62,96.9 \%)$, Both lungs involvement $(61,95.3 \%)$, Ground-glass opacity $(48,75 \%)$, and Consolidation $(42,65.6 \%)$. In most lights, there were cloud turbulence and ground-glass high density scattered in the periphery of both 
lungs, local patchy consolidation and Broncho pneumatic signs were seen. The severe and critical cases had more diffuse density, solid or stripy lesion with local fibrosis formation in the lower leaves of lungs (Figure 4). Up to 45 cases had patchy high density in more than 3 leaves.

\section{Electrocardiography:}

To evaluate the function of heart lesion, we also did the electrocardiography examination (Data not shown). 44 cases were normal, 10 were sinus rhythm and t-wave changed, 4 had high voltage in the left ventricle with sinus rhythm, 2 nodal tachycardia, 2 left anterior fascicular block with sinus rhythm, 2 t-wave changed accompanied with U-wave elevated and sinus rhythm.

\section{The progress of disease:}

In our studies, the temperature of fevers returned to normal by treatment in 1-5 days (2.7 days as average). By closing obvious every three days, we could see the tendency of the indexes change during treatment. The key indexes, LDH and LC may keep progress to extreme point on the 3 days after hospitalization (6-9 days after onset) (Figure 5) . Corrected electrolyte imbalance and kept an eye on potassium was important.

All 64 cases were treated by triple therapy (Interferon, Lopinavir/ritonavir, Abidor),9 (14.06\%) had glucocorticoid treated, and $19(29.69 \%)$ had antibiotic use with 8 days as the average course. The average of hospitalization was 25.8 days, all patients were discharged by data were collected (March 4, 2020) (Table 1 ). The COVID-19 nucleic acid test transforming to negative was about 10-20 days (12.8 as average).

\section{Discussion}

To help further understand COVID-19 infection and treatment, we studied 64 cases in Wenzhou. The epidemic information showed obviously that COVID-19 could spread from person to person, having family and community aggregation, which may cause public transmission. The result was in keeping with a familial cluster report [16]. We have 25 cases of Gen.2, a little less than Gen.1 (33 cases), which meant Wenzhou were still facing the press of importing cases, and the epidemic of local was still a potential threat that needed to pay attention. Regardless, the number of cases increase slowly donated to the strategy of city blockade that made by the government from Jan $25^{\text {th }}$ and Feb $2^{\text {nd }}\left[\begin{array}{lll}17 & 18\end{array}\right]$.

In our cases, the majority cases $(58,90.63 \%)$ were ranged from $20-60$ (median age was 44 ), few $(5,7.81 \%$ ) were above 60. It was inconsistent with the cases reported in Wuhan[19 20], but similar to Korea[21]. The reasons were various: the aged had low immunity compared to the youngers and Wuhan had a great number of elders; the breakout of COVID-19 in short time challenged the hospital resource, while the cases we had in Wenzhou were imported by travelers transmitted from Wuhan or spread by close contacting to them, the main of those were young and middle aged; genomic and infectivity of virus may evolve after outbreak; furthermore, we may not be clear to the virus in early phase for information lacking. Notably, we reported the minimum age of patient with 26-months old, who got virus from mother, the pre-minimum age was reported as 6 months [22]. Thus, the whole population may be susceptible to COVID-19.

There were 51 light cases, 11 severity and 2 critical in 64 cases, no death. About half had basic disease, especially hypertension; the elders or with basic disease may have more serious conditions. Most cases (59, 92.19\%) had fever, the average temperature was 38.1. Temperature was shown to have correlation to disease severity. Respiratory symptoms were seen in 45 cases (70.3\%), as 28 (43.8\%) had gastrointestinal symptoms. Above half $(39,60.9 \%)$ had Weakness, few $(5,7.8 \%)$ had muscular soreness. Importantly, 5 cases had no fever, just with mild symptoms like cough, pharyngalgia, stuffiness or nausea, but PCR tests were positive. One case even did not have any symptom. Among these 5 cases, 3 were definite as Gen.1, 2 were Gen.2, the latency of them ranged from 1-14. This important information indicted that the COVID-19 could be infected with no symptoms, and the latency of COVID-19 could be up to 14 days without showing any typical or obvious symptoms. COVID-19 was so craft that it may conceal in health groups, and had much more infectivity than SARS, it may probably cause pandemic, infecting more population than SARS absolutely.

All 64 cases were tested to be PCR positive in an interval more than $24 \mathrm{~h}$ in respiratory samples (sputum or 
throat swabs). Although nucleic acid of PCR test had high specificity, due to the unclear course of infection and skill required to take swab samples, the sensitivity was not satisfied with only $30-40 \%$ in suspect patients $(47.4 \%, 9 / 19)[23]$. But it was showed by the lab information that the virus copies could be tested to reach highest at 7th day after disease onset. It was reported that in diagnosed cases, the virus could also be detected in $10 \%$ patients' blood samples at acute period and 50\% of feces[24]. Another report showed 88.9\% (8/9) stool sensitivity while blood and urine were negative[23]. Saliva also had $91.7 \%(11 / 12)$ positive in diagnostic patients[25]. The PCR positive rate was highly depending on the sample quality and course of infection. Another problem was the safety. The doctor faced high infectivity risk during obtaining sample. And PCR test have its longtime cost disadvantage as well. Compared to PCR, chest CT was much more convenient and safety. In our study, 62 cases had varying degrees of lung symptoms by checking with chest CT, Pneumonia (62, 96.9\%), Both lungs involvement (61,95.3\%), Ground-glass opacity (48, 75\%), and Consolidation (42, $65.6 \%$ ). One of two cases having no lung changes in CTs but had high fever as 38; the other one did not show any symptoms. Cases with normal CT and PCR positive had also been reported[26 27]. Thus, chest $\mathrm{CT}$ and PCR of nucleic acid shall be combined considered in clinical high suspicion. Besides, a few patients had rhythm and wave changed in their electrocardiography that may be caused by low potassium ion under the attack of virus.

It was also found that patients may have many lower indexes including WBC, LC, PLT, K+, Na+, OI, and high LDH, CRP, D-dimer (Figure 2 ), BNP and TNI were normal, consistent with a report of multicenter study[26]. Correlation analyzation showed that the severity had positive correlated to age, temperate, LDH, D-dimer and negative to LC and OI (Table 3, Figure 3 ). LDH and LC were good biomarkers for monitoring progress of disease in all classifications (Figure 5 ). The low potassium may be caused by feed less when fevering or vomiting, diarrhea by antivirus drug use, and would have influence with weakness, dyspnea. Thus, it was important to correct low potassium and keep a close obvious on it during treatment.

The average of hospital stay was 25.8 days, the temperature of fevers returned to normal with treatment in 1-5 days (2.7 days as average), 20 were discharged when data were collected. The average of COVID-19 nucleic acid test transforming to negative was 12.8 days. In early phase, the infected lungs presented changes like ground-glass, cloud turbulence or paving stone pattern, then developed as consolidation within more than 2 leaves later, having local pulmonary fibrosis if deteriorated. The inflammation could be absorbed in about 7-10 days if treated well. The triple therapy (Interferon, Lopinavir/ritonavir, Abidor) was confirmed to be effective in treating COVID-19 infection. Weakness and diarrhea during treatment may be related to virus attack or Lopinavir/ritonavir taken.

ACE2, Angiotensin I Converting Enzyme 2, was confirmed to be the cell entry receptor of COVID-19 as SARS-CoV[28]. The protein was expressed in endothelial cells, arterial smooth muscle cells, lung alveolar epithelial cells, lymphocyte, heart, kidney, testis, ovary, and gastrointestinal system[29]. It explained why COVID-19 could be detected in samples of respiratory and gastrointestinal system and blood as SARS[2] and lymphopenia caused. It also reminded us COVID-19 may have influence on sterility as SARS, needed to call attention. High expression of ACE2 was also related to hypertension and smoke, accord with the vulnerable and high mortality to elders, smokers and those who had basic disease of hypertension. The protein was involved in regulating of calcium-activated potassium channel activity by related to CALM1, CALM3, CHRNA9 and CHARNA10 (String Database), leading to low potassium in patients infected with COVID-19 and SARS, that may result in weakness, dyspnea and rhythm.

Due to close relative genomic and same virus receptor ACE2, COVID-19 had similarity clinical features to SARS, such as fever, high temperate, respiratory and gastrointestinal system mainly involved, similar chest CT change, low potassium, low LC, low OI, high LDH and so on. Most of infection were light cases, suggested that it was milder than SARS. But had much stronger infectivity, with high infectivity when in latency without any symptoms. Early diagnosis and treatment when symptom showed, would bring it a better outcome. We shall do a good job in public health management, strengthen health publicity and education to control further spread of the epidemic as possible. With the experience in handling SARS, we have confidence and do will defeat the COVID-19 in the end. 


\section{Reference:}

1. Richman DD WR, Hayden FG. Clinical virology . 4th ed. Washington: ASM Press, 2016.

2. Drosten C, Gunther S, Preiser W, et al. Identification of a novel coronavirus in patients with severe acute respiratory syndrome. N Engl J Med 2003;348 (20):1967-76 doi: 10.1056/NEJMoa030747[published Online First: Epub Date]|.

3. Ksiazek TG, Erdman D, Goldsmith CS, et al. A novel coronavirus associated with severe acute respiratory syndrome. N Engl J Med 2003;348 (20):1953-66 doi: 10.1056/NEJMoa030781[published Online First: Epub Date]|.

4. Kuiken T, Fouchier RA, Schutten M, et al. Newly discovered coronavirus as the primary cause of severe acute respiratory syndrome. Lancet 2003;362 (9380):263-70 doi: 10.1016/S0140-6736(03)13967-0[published Online First: Epub Date]|.

5. Zaki AM, van Boheemen S, Bestebroer TM, Osterhaus AD, Fouchier RA. Isolation of a novel coronavirus from a man with pneumonia in Saudi Arabia. N Engl J Med 2012;367 (19):1814-20 doi: 10.1056/NEJMoa1211721[published Online First: Epub Date]|.

6. de Groot RJ, Baker SC, Baric RS, et al. Middle East respiratory syndrome coronavirus (MERS-CoV): announcement of the Coronavirus Study Group. J Virol 2013;87 (14):7790-2 doi: 10.1128/JVI.0124413[published Online First: Epub Date]|.

7. 高艳梅, 魏芳. 细胞凋亡抑制蛋白-1在宫颈癌及宫颈癌化疗耐药方面的研究进展. 中华临床医师杂志 (电子 版) (4)

8. PD M, S S, CL D. Assembling the building blocks: structure and function of inhibitor of apoptosis proteins. Cell death and differentiation 2010;17 (1):46-53 doi: 10.1038/cdd.2009.45[published Online First: Epub Date]|.

9. Lacasse EC, Baird S, Korneluk RG, MacKenzie AE. The inhibitors of apoptosis (IAPs) and their emerging role in cancer.17 (25):3247-59

10. Deveraux QL, Takahashi R, Salvesen GS, Reed JC. X-Linked IAP is a direct inhibitor of cell-death proteases. Nature 1997;388 (6639):300-04

11. Chai J, Du C, Wu JW, Kyin S, Shi Y. Structural and biochemical basis of apoptotic activation by Smac/DIABLO. Nature 2000;406 (6798):855-62

12. Yu W-BT, Guang-Da ;Zhang, Li ; Corlett, Richard T. . Decoding the evolution and transmissions of the novel pneumonia coronavirus (SARS-CoV-2) using whole genomic data.

13. Coronavirus disease (COVID-19) Situation dashboard: WHO., 2020.

14. China NHCotPsRo. Diagnosis and treatment of pneumonia caused by novel coronavirus infection (Trial version 5), 2020.

15. China NHCotPsRo. Diagnosis and treatment of severe and critical cases of new coronavirus pneumonia (Trial version 2), 2020.

16. Chan JF, Yuan S, Kok KH, et al. A familial cluster of pneumonia associated with the 2019 novel coronavirus indicating person-to-person transmission: a study of a family cluster. Lancet 2020;395 (10223):514-23 doi: 10.1016/S0140-6736(20)30154-9[published Online First: Epub Date]|.

17. Notice of leading group for prevention and control of pneumonia caused by new coronavirus infection in wenzhou city (No.1). In: city Lgfpacopcbnciiw, ed. Wenzhou: Wenzhou daily nespaper, 2020.

18. Notice of leading group for prevention and control of pneumonia caused by new coronavirus infection in wenzhou city (No.6). In: city Lgfpacopcbnciiw, ed. Wenzhou: Wenzhou daily nespaper, 2020. 
19. Wang D, Hu B, Hu C, et al. Clinical Characteristics of 138 Hospitalized Patients With 2019 Novel Coronavirus-Infected Pneumonia in Wuhan, China. JAMA 2020 doi: 10.1001/jama.2020.1585[published Online First: Epub Date]|.

20. Chen N, Zhou M, Dong X, et al. Epidemiological and clinical characteristics of 99 cases of 2019 novel coronavirus pneumonia in Wuhan, China: a descriptive study. Lancet 2020;395 (10223):507-13 doi: 10.1016/S0140-6736(20)30211-7[published Online First: Epub Date]|.

21. Ki M, nCo VT. Epidemiologic characteristics of early cases with 2019 novel coronavirus (2019-nCoV) disease in Republic of Korea. Epidemiol Health 2020:e2020007 doi: 10.4178/epih.e2020007[published Online First: Epub Date]|.

22. Wang D, Ju XL, Xie F, et al. [Clinical analysis of 31 cases of 2019 novel coronavirus infection in children from six provinces (autonomous region) of northern China]. Zhonghua Er Ke Za Zhi 2020;58 (4):E011 doi: 10.3760/cma.j.cn112140-20200225-00138[published Online First: Epub Date]|.

23. Xie C, Jiang L, Huang G, et al. Comparison of different samples for 2019 novel coronavirus detection by nucleic acid amplification tests. Int J Infect Dis 2020 doi: 10.1016/j.ijid.2020.02.050[published Online First: Epub Date]|.

24. Xu K, Cai H, Shen Y, et al. [Management of corona virus disease-19 (COVID-19): the Zhejiang experience]. Zhejiang Da Xue Xue Bao Yi Xue Ban 2020;49 (1):0

25. To KK, Tsang OT, Chik-Yan Yip C, et al. Consistent detection of 2019 novel coronavirus in saliva. Clin Infect Dis 2020 doi: 10.1093/cid/ciaa149[published Online First: Epub Date]|.

26. Yang W, Cao Q, Qin L, et al. Clinical characteristics and imaging manifestations of the 2019 novel coronavirus disease (COVID-19):A multi-center study in Wenzhou city, Zhejiang, China. J Infect 2020 doi: 10.1016/j.jinf.2020.02.016[published Online First: Epub Date]|.

27. Xu YH, Dong JH, An WM, et al. Clinical and computed tomographic imaging features of Novel Coronavirus Pneumonia caused by SARS-CoV-2. J Infect 2020 doi: 10.1016/j.jinf.2020.02.017[published Online First: Epub Date]|.

28. Zhou P, Yang XL, Wang XG, et al. A pneumonia outbreak associated with a new coronavirus of probable bat origin. Nature 2020 doi: 10.1038/s41586-020-2012-7[published Online First: Epub Date]|.

29. mRNA Expression by UniProt/SwissProt for ACE2 Gene: GeneCards, 2020.

Table

Table 1 Basic information and clinical features of 64 cases of COVID-19 in Wenzhou Characteristics

Total

Table 1 Basic information and clin Value

Age(year) Median

20

$20-60$

60

Gender

Male

64

Female

44 ? ¿?

Generation

$1 / 64(1.56 \%)$

$58 / 64(90.63 \%) ? ¿$ ?

$5 / 64(7.81 \%)$

1 (been to Wuhan in last 14 days)

$36 / 64(56.25 \%)$

$28 / 64(43.75 \%)$

2 (close contact to 1$)$

3 (close contact to 2 )

$33 / 64(51.56 \%)$

$25 / 64(39.06 \%)$

$6 / 64(9.38 \%)$

Severity 
Light

Severe

Critical

Latency(62 cases had close contact history)(days)

Mean of latency

Longest latency

Shortest latency

Discharge (till Feb 14)

Of Light

Of Severe

Of Critical

Average hospital stay(day)

Triple therapy (Interferon, Lopinavir/ritonavir, Abidor)

Glucocorticoid

Antibiotic use

Average of course of antibiotic use(days)

Average of PCR transform to negative(days)

Temperature () (Mean \pm SD)

Fever

Weakness

Muscular soreness

Lung Symptoms (by Chest Computer Tomographic)

Pneumonia

Lungs involvement (Both)

Ground-glass opacity

Consolidation

Respiratory Symptoms

Dry Cough

Cough and expectoration

Shortness of breath

Pharyngalgia

Bloody phlegm

Nasal obstruction

Yellow spectrum

Gastrointestinal symptoms

Diarrhea

Nausea

Vomit

No symptoms (including no fever)

Secondary infection

Basic disease

Smoke

Hypertension

Chronic Liver disease
$51 / 64(79.69 \%)$

$11 / 64(17.19 \%)$

$2 / 64(3.12 \%)$

Latency (62 cases had close contact 3.44

14

1

20

$13 / 51(25.49 \%)$

$6 / 11(54.55 \%)$

$1 / 2(50 \%)$

21.1

8 days/course

$9 / 64(14.06 \%)$

$19 / 64(29.69 \%)$

8

12.8

$38.1 \pm 0.85^{* *}$

$59 / 64(92.19 \%)$

$39 / 64(60.9 \%)$

$5 / 64(7.8 \%)$

$62 / 64(96.9 \%)$

62

61

48

42

$45 / 64(70.3 \%)$

41

23

18

10

4

4

3

$28 / 64(43.8 \%)$

14

13

8

15

$27 / 64$

None

$20 / 64$

7

** $\mathrm{p}<0.001$ 


\begin{tabular}{|c|c|c|c|c|c|c|c|}
\hline Table 2 & Table 2 & Table 2 & Table 2 & Table 2 & Table 2 & Table 2 & \\
\hline Clinical & Clinical & Clinical & Clinical & Clinical & Clinical & Clinical & \\
\hline indexes of & indexes of & indexes of & indexes of & indexes of & indexes of & indexes of & \\
\hline 64 cases of & 64 cases of & 64 cases of & 64 cases of & 64 cases of & 64 cases of & 64 cases of & \\
\hline $\begin{array}{l}\text { COVID-19 } \\
\text { in }\end{array}$ & $\begin{array}{l}\text { COVID-19 } \\
\text { in }\end{array}$ & $\begin{array}{l}\text { COVID-19 } \\
\text { in }\end{array}$ & $\begin{array}{l}\text { COVID-19 } \\
\text { in }\end{array}$ & $\begin{array}{l}\text { COVID-19 } \\
\text { in }\end{array}$ & $\begin{array}{l}\text { COVID-19 } \\
\text { in }\end{array}$ & $\begin{array}{l}\text { COVID-19 } \\
\text { in }\end{array}$ & \\
\hline Wenzhou & Wenzhou & Wenzhou & Wenzhou & Wenzhou & Wenzhou & Wenzhou & \\
\hline & Minimum & Maximum & Mean & $\mathrm{SD}$ & $\begin{array}{l}\text { Normal } \\
\text { Reference } \\
(95 \% \text { CI })\end{array}$ & $\mathrm{P}$ value & $\mathrm{P}$ value \\
\hline $\begin{array}{l}\text { WBC } \\
\left(10^{\wedge} 9 / \mathrm{L}\right)\end{array}$ & 2.20 & 9.20 & 4.65 & 1.65 & $3.5-9.5$ & $<0.001$ & $<0.001$ \\
\hline $\begin{array}{l}\text { NEC } \\
\left(10^{\wedge} 9 / \mathrm{L}\right)\end{array}$ & 1.30 & 7.60 & 3.08 & 1.35 & $1.8-6.3$ & $<0.001$ & $<0.001$ \\
\hline $\begin{array}{l}\mathrm{LC} \\
\left(10^{\wedge} 9 / \mathrm{L}\right)\end{array}$ & 0.30 & 3.10 & 1.16 & 0.55 & $1.1-3.2$ & $<0.001$ & $<0.001$ \\
\hline $\begin{array}{l}\text { PLT } \\
\left(10^{\wedge} 9 / \mathrm{L}\right)\end{array}$ & 94.00 & 430.00 & 186.89 & 71.29 & $125-350$ & $<0.001$ & $<0.001$ \\
\hline \multirow[t]{2}{*}{$\mathrm{Hb}(\mathrm{g} / \mathrm{L})^{* *}$} & 116 & 162 & $\begin{array}{l}\text { Male } \\
140.9\end{array}$ & 11.34 & $135-170$ & $<0.001$ & $<0.001$ \\
\hline & 98 & 158 & Female129.8 & 12.73 & $115-150$ & 0.275 & 0.275 \\
\hline $\operatorname{ALT}(\mathrm{U} / \mathrm{L})$ & 5.00 & 206.00 & 30.55 & 29.88 & $7-40$ & 0.064 & 0.064 \\
\hline $\operatorname{AST}(\mathrm{U} / \mathrm{L})^{*}$ & 6.00 & 142.00 & 31.78 & 19.95 & $13-35$ & 0.003 & 0.003 \\
\hline $\mathrm{K}^{+}(\mathrm{mmol} / \mathrm{L})^{* *}$ & 2.68 & 5.05 & 3.65 & 0.38 & $3.5-5.3$ & $<0.001$ & $<0.001$ \\
\hline $\mathrm{Na}^{+}(\mathrm{mmol} / \mathrm{L})^{*}$ & *** 127.70 & 140.50 & 136.55 & 2.59 & $137-147$ & $<0.001$ & $<0.001$ \\
\hline $\operatorname{Cr}(\mu \mathrm{mol} / \mathrm{L})$ & 24.00 & 126.00 & 68.28 & 18.25 & $38-94$ & 0.321 & 0.321 \\
\hline $\mathrm{CK}(\mathrm{U} / \mathrm{L})$ & 28.00 & 1522.00 & 150.28 & 244.68 & $40-200$ & 0.326 & 0.326 \\
\hline $\mathrm{LDH}(\mathrm{U} / \mathrm{L})^{* *}$ & 87.00 & 455.00 & 222.88 & 80.37 & $120-250$ & $<0.001$ & $<0.001$ \\
\hline $\begin{array}{l}\text { TNI } \\
(\mathrm{ng} / \mathrm{ml})\end{array}$ & 0.02 & 0.09 & 0.03 & 0.02 & $<0.1$ & 0.877 & 0.877 \\
\hline $\begin{array}{l}\mathrm{BNP} \\
(\mathrm{ng} / \mathrm{L})\end{array}$ & 100 & 434 & 108.13 & 45.85 & $<300$ & 0.161 & 0.161 \\
\hline $\operatorname{CRP}(\mathrm{mg} / \mathrm{L})^{* *}$ & 0.60 & 117.00 & 22.76 & 24.46 & $<10$ & $<0.001$ & $<0.001$ \\
\hline$\underset{* *}{\mathrm{OI}}(\mathrm{mmHg})$ & 123 & 714 & 378.08 & 119.16 & $400-500$ & $<0.001$ & $<0.001$ \\
\hline $\begin{array}{l}\text { D-dimer } \\
(\mu \mathrm{g} / \mathrm{L})^{* *}\end{array}$ & 59 & 747 & 182.42 & 143.18 & $<200$ & $<0.001$ & $<0.001$ \\
\hline
\end{tabular}

** $\mathrm{p}<0.001,{ }^{*} \mathrm{p}<0.05$

Table 3 Pearson's Correlation analyzation of clinical features and indexes Table 3 Pearson's Correlation a

Severity

R2

Age

$\mathrm{P}$ value

R2

$\mathrm{P}$ value 
Temper

LC

LDH

Fer

D-dimer

OI

*. $\mathrm{P}<0.05$ (bilateral)

**. $\mathrm{P}<0$.01 (bilateral)

R2

$\mathrm{P}$ value

R2

$\mathrm{P}$ value

R2

$P$ value

R2

$P$ value

R2

$\mathrm{P}$ value

R2

$P$ value

*. $\mathrm{P}<0.05$ (bilateral)

**. $\mathrm{P}<0.01$ (bilateral)

\section{Figure legends:}

Fig.1 Basic information and clinical features of 64 cases of COVID-19 in Wenzhou In age, $1(1.56 \%)$ under $20,58(90.63 \%)$ from $20-60,5(7.81 \%)$ above 60 ; in gender, $36(56.25 \%)$ were male and $28(43.75 \%)$ were female; in generation, 33 persons were classed as Generation 1 (Gen.1); 25 cases as Gen.2; 4 cases as Gen.3; 2 cases lost contact history; in severity classification, 51 cases light, 11 severity and 2 critical.

Fig.2 Clinical indexes of 64 cases of COVID-19 in Wenzhou WBC, NEC, LC, PLT, Hb, K+, $\mathrm{Na}+$, LDH, CRP, OI (Oxygenation Index) and D-dimer had statistically significant $(\mathrm{p}<0.001)$, so did $\operatorname{AST}(\mathrm{p}<0.05)$. The indexes decreased in patients infected with COVID-19 including WBC, LC, PLT, K+, $\mathrm{Na}+$, OI, while LDH, CRP, D-dimer would be at higher level. BNP and TNI were normal, and not shown.

Fig.3 The distribution of data of indexes correlated to disease severity Severity had the positive correlation with D-dimer, and age, temperate, LDH, while had negative correlation with LC and OI.

Fig.4 CT changes between primary diagnostic with before discharged in vary degrees of COVID-19 infection There were cloud turbulence and ground-glass high density scattered in the periphery of both lungs in most light cases; local patchy consolidation and Broncho pneumatic signs could be seen. The severe cases had more diffuse density, solid or stripy lesion as well as local fibrosis formation in the lower leaves of lungs.

Fig.5 Close obvious of LDH $\backslash \mathbf{L C} \backslash \mathrm{K}+$ changes during hospitalization in vary degrees of COVID19 infectionLC, $\mathrm{LDH}$ and $\mathrm{K}+$ could be good biomarkers to predicting and monitoring the progress of disease. 

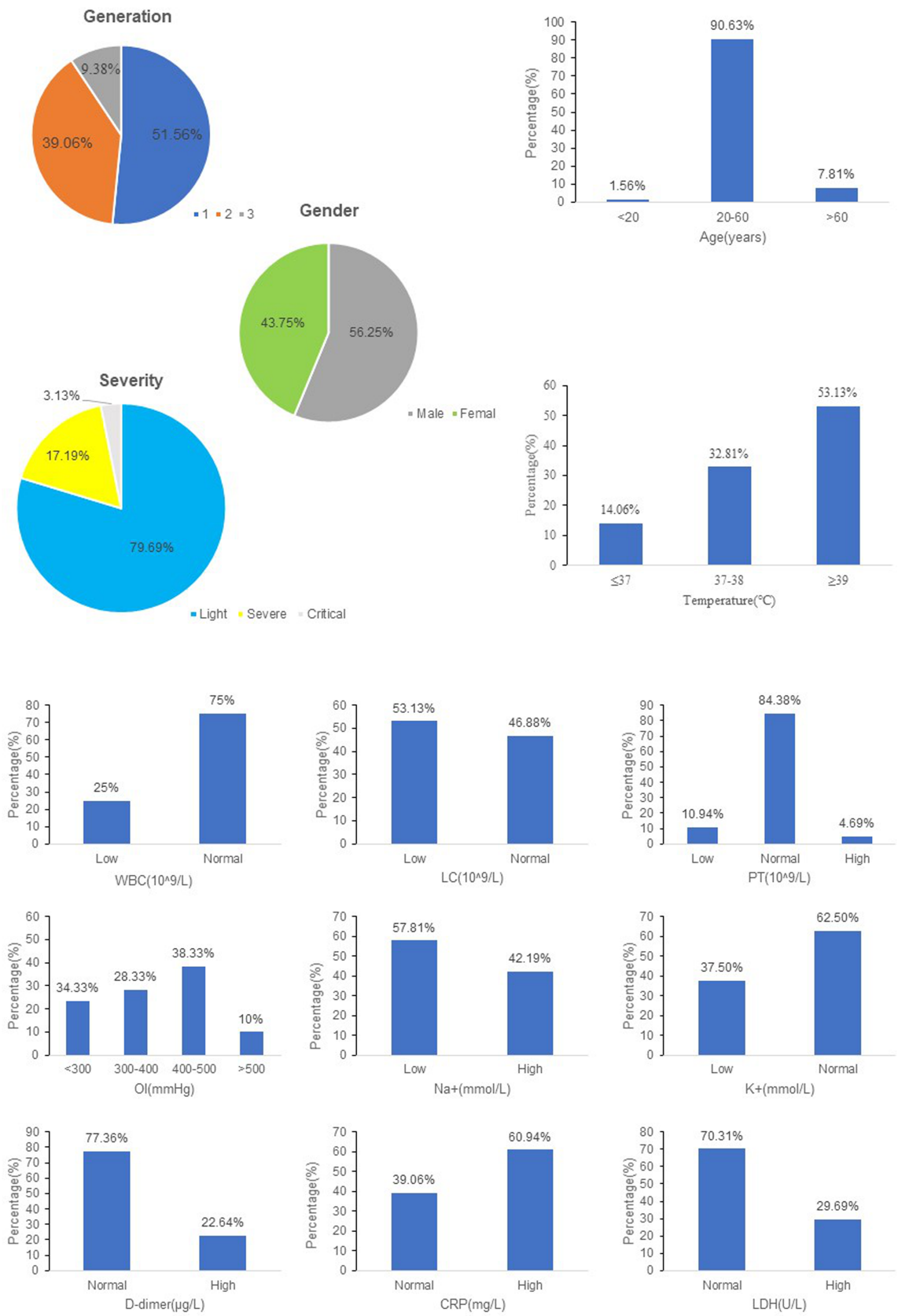

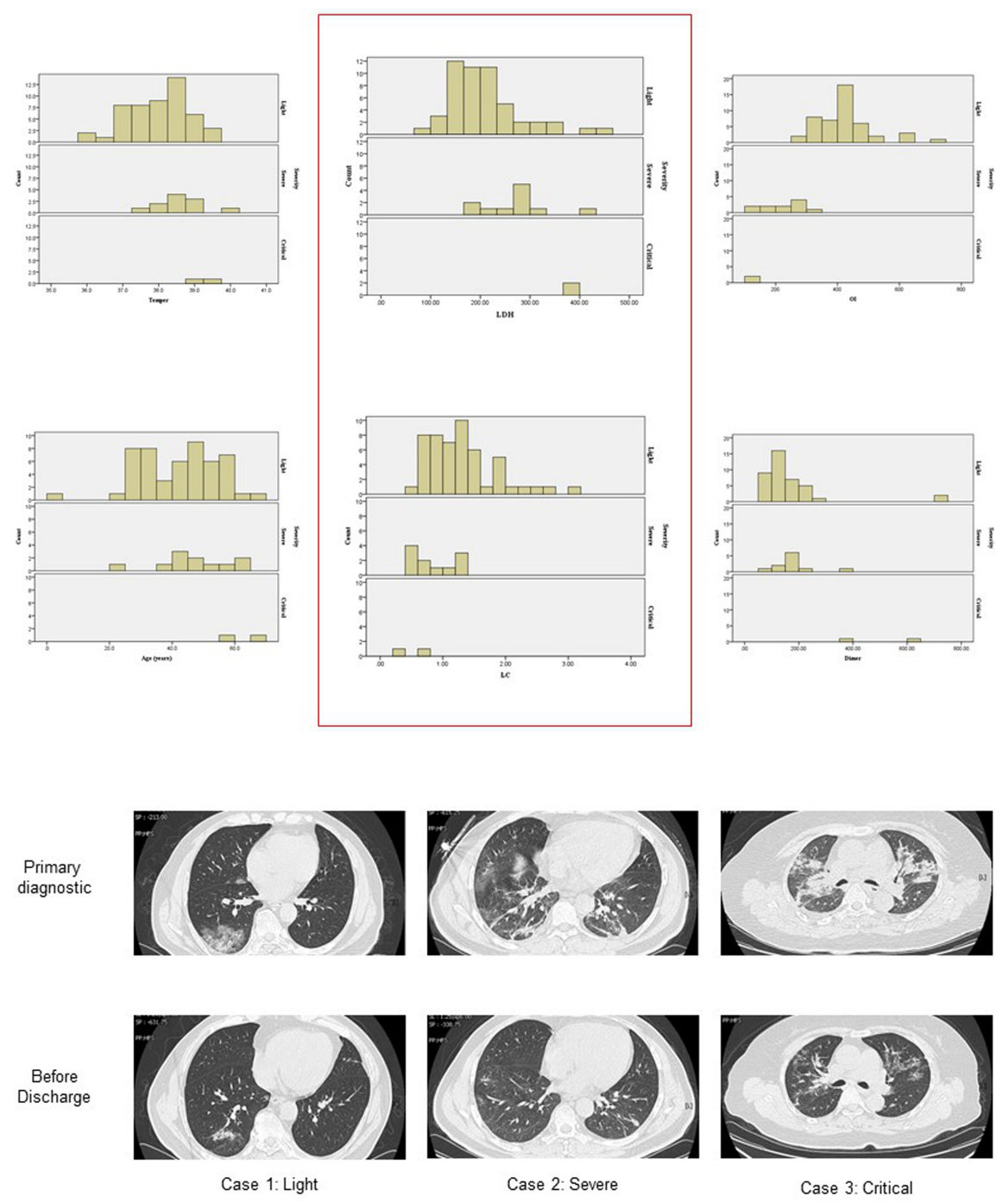
LDH $(\mathrm{U} / \mathrm{L})$

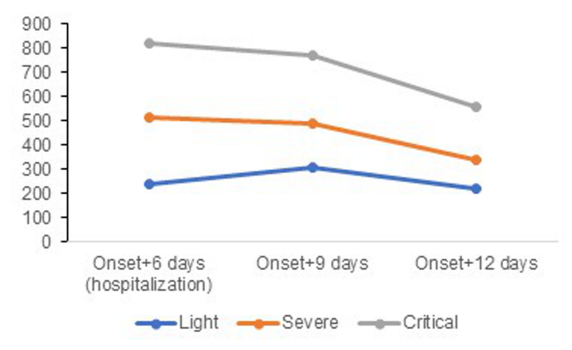

LC (10^9/L)

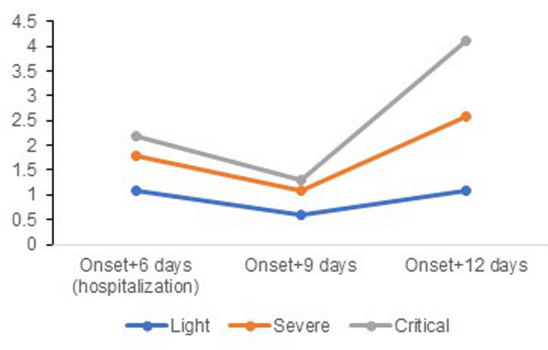

$\mathrm{K}+(\mathrm{mmol} / \mathrm{L})$

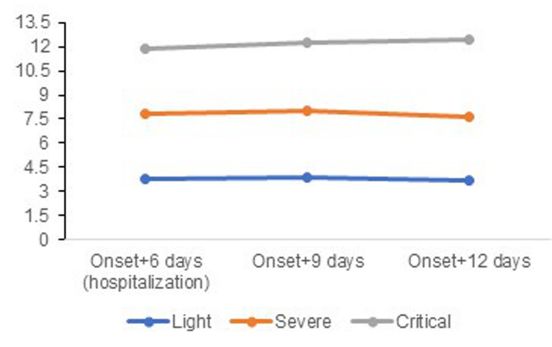

